\title{
Use of parenteral nutrition in general and surgical pediatric wards: indications and complications
}

Francesca Penagini ${ }^{1}$, Luciano Maestri ${ }^{2}$, Giovanna Zuin ${ }^{1}$,Giulia Ramponi ${ }^{1}$, Barbara Borsani ${ }^{1}$, Federico Rebosio ${ }^{2}$, Giovanna Riccipetitoni ${ }^{2}$, Gian Vincenzo Zuccotti ${ }^{1}$.

${ }^{1}$ Clinica Pediatrica, Ospedale dei Bambini V. Buzzi, Università degli Studi di Milano.

${ }^{2}$ Chirurgia Pediatrica, Ospedale dei Bambini V. Buzzi, Università degli Studi di Milano

Sistema Socio Sanitario

Regione

Lombardia

ASST Fatebenefratelli Sacco
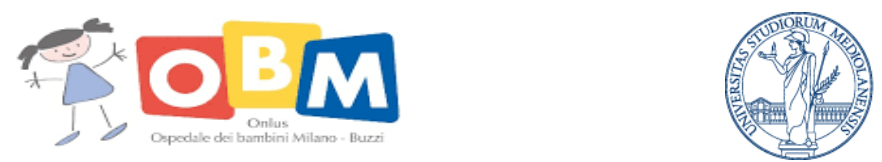

UNIVERSITÀ

DEGLI STUDI

DI MILANO

\section{Introduction}

Parenteral nutrition (PN) is used to treat children that cannot fully feed by oral or enteral route. Nutrition support with PN in childhood is highly variable depending on the age and pathology of inpatient.

\section{Aim}

The aim of our retrospective study was to evaluate the current clinical practice concerning the use of PN in our General Pediatric and Surgical Wards.

\section{Materials and Methods}

Clinical records of inpatients admitted on the General and Surgical Pediatric Wards from January 2012 to December 2016 were retrospectively analyzed. Indications for PN, route of administration and complications were evaluated.

\section{Results}

From 2012 to 2016: 21 patients on the General Pediatric Ward and 292 patients on the Surgical Pediatric Ward required PN. In all cases PN was administered via internal jugular vein or a Broviac line.

On General Pediatric Ward, the most frequent indication for PN was inflammatory bowel disease (IBD), followed by malnutrition in children with anatomical malformations, neurological patients and metabolic diseases. Figure 1. Mean duration was $11,46+17,37$ days.

On Pediatric Surgical Ward, the most frequent indications for PN were..... Mean duration was $9,6+10,9$ days.
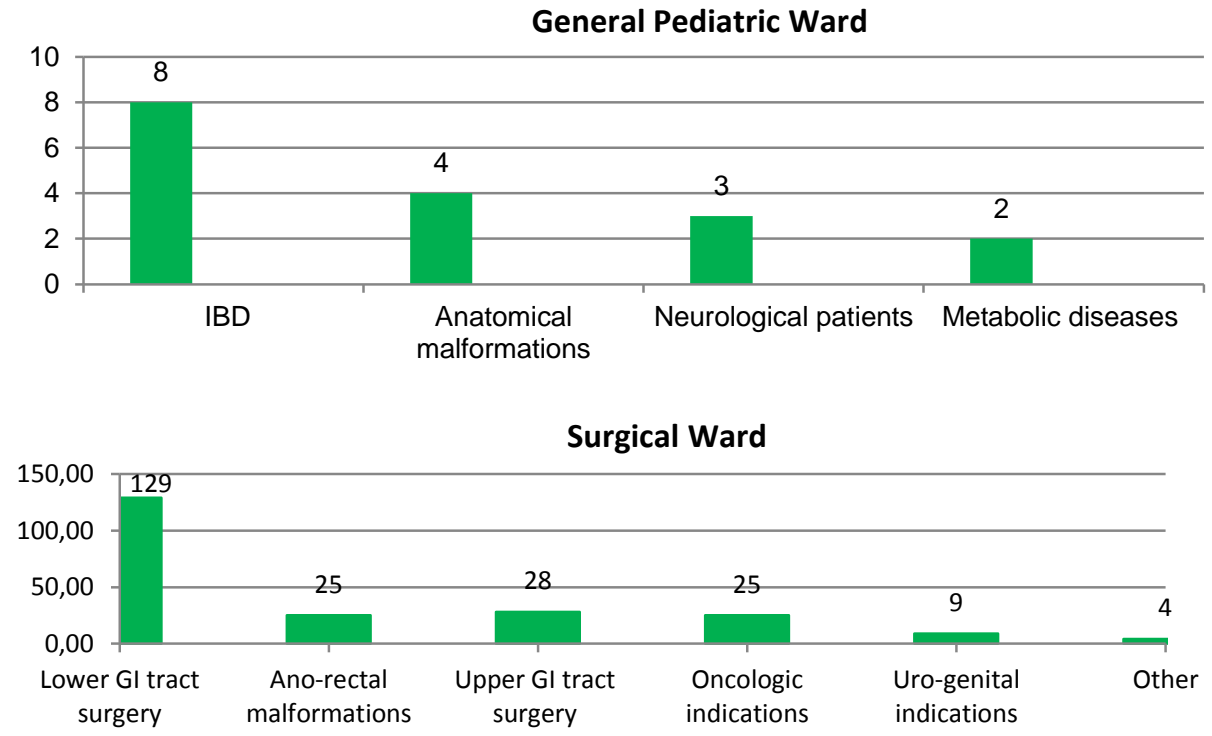

Conclusions

PN has been safely used for a wide range of indications and with a low rate of complications. 